\title{
Heterogeneous materials: a new class of materials with unprecedented mechanical properties
}

\section{Xiaolei Wu \& Yuntian Zhu}

To cite this article: Xiaolei Wu \& Yuntian Zhu (2017): Heterogeneous materials: a new class of materials with unprecedented mechanical properties, Materials Research Letters, DOI: 10.1080/21663831.2017.1343208

To link to this article: http://dx.doi.org/10.1080/21663831.2017.1343208
(c) 2017 The Author(s). Published by Informa UK Limited, trading as Taylor \& Francis Group.

\section{Published online: 26 Jun 2017.}

6 Submit your article to this journal 4

\section{Q View related articles $\square$}

View Crossmark data ¿ 


\title{
Heterogeneous materials: a new class of materials with unprecedented mechanical properties
}

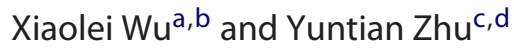

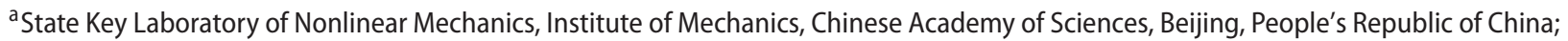 \\ ${ }^{b}$ School of Engineering Science, University of Chinese Academy of Sciences, Beijing, People's Republic of China; ${ }^{\circ}$ Department of Materials \\ Science and Engineering, North Carolina State University, Raleigh, NC, USA; ${ }^{d}$ Nano Structural Materials Center, School of Materials Science and \\ Engineering, Nanjing University of Science and Technology, Nanjing, People's Republic of China
}

\begin{abstract}
Here we present a perspective on heterogeneous materials, a new class of materials possessing superior combinations of strength and ductility that are not accessible to their homogeneous counterparts. Heterogeneous materials consist of domains with dramatic strength differences. The domain sizes may vary in the range of micrometers to millimeters. Large strain gradients near domain interfaces are produced during deformation, which produces a significant back-stress to strengthen the material and to produce high back-stress work hardening for good ductility. High interface density is required to maximize the back-stress, which is a new strengthening mechanism for improving mechanical properties.
\end{abstract}

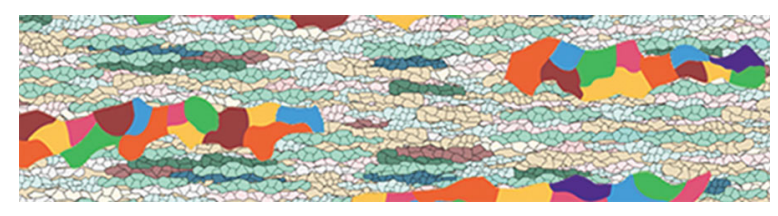

IMPACT STATEMENT

Heterogeneous materials are becoming the next hot research field after the nanomaterials era.

\section{ARTICLE HISTORY}

Received 27 May 2017

\section{KEYWORDS}

Heterogeneous materials; strain gradient; back-stress; mechanical incompatibility; mechanical properties

\section{Background}

Materials are either strong or ductile, but rarely both at the same time $[1,2]$. Stronger and tougher materials are desired for many structural applications such as transportation vehicles for higher energy efficiency and better performance. For the last three decades, nanostructured (ultrafine-grained) metals have been extensively studied because of their high strength. However, overcoming their low ductility has been a challenge [2-25], which is one of the major reasons why they have not been widely commercialized for industrial applications. Another major obstacle to practical structural applications of nanostructured metals is the challenge in scaling up for industrial production at low cost [26].
After over a century's research, we have almost reached the limit on how much further we can improve the mechanical properties of metals and alloys. Our conventional wisdom from the textbook and literature is to reinforce a weak matrix by a stronger reinforcement such as second-phase particles or fibers. A question arises on if there exist yet-to-be-explored new strategies to make the next generation of metals and alloys with a 'quantum jump' in strength and ductility instead of the incremental improvements that we have seen for the last several decades.

Recently, there have been several reports on superior combinations of strength and ductility in various metals and alloys that are processed to have widely dif-

CONTACT Xiaolei Wu xlwu@imech.ac.cn $\Theta$ State Key Laboratory of Nonlinear Mechanics, Institute of Mechanics, Chinese Academy of Sciences, 15 Beisihuan West Road, Beijing 100190, People's Republic of China; Yuntian Zhu ytzhu@ncsu.edu Department of Materials Science and Engineering, North Carolina State University, Raleigh, NC 27695, USA; Nano Structural Materials Center, School of Materials Science and Engineering, Nanjing University of Science and Technology, 200 Xiaolingwei, Nanjing 210094, People's Republic of China 
ferent microstructures, including the gradient structure [27-32], heterogeneous lamella structure [33], bimodal structure [10,25,34,35], harmonic structure [36-38], laminate structure [39,40], dual-phase steel [41-43], nanodomained structure [44], nanotwinned grains [45,46], etc. [47]. These materials have one common feature: there is a dramatic difference in strength between different domains, while the sizes and geometry of the domains may vary widely. In other words, there are huge microstructural heterogeneities in these materials. Therefore, these materials can be considered as heterogeneous materials.

In this perspective, we will present the fundamental physics that renders these heterogeneous materials superior mechanical properties as well as the microstructures that are required for producing the best mechanical properties.

\section{Definition of heterogeneous materials}

Heterogeneous materials can be defined as materials with dramatic heterogeneity in strength from one domain area to another. This strength heterogeneity can be caused by microstructural heterogeneity, crystal structure heterogeneity or compositional heterogeneity. The domain sizes could be in the range of micrometers to millimeters, and the domain geometry can vary to form very diverse material systems.

\section{Deformation behavior of heterogeneous materials}

During deformation, for example, tensile testing, of the heterogeneous materials, with increasing applied strain, the deformation process can be classified into three stages

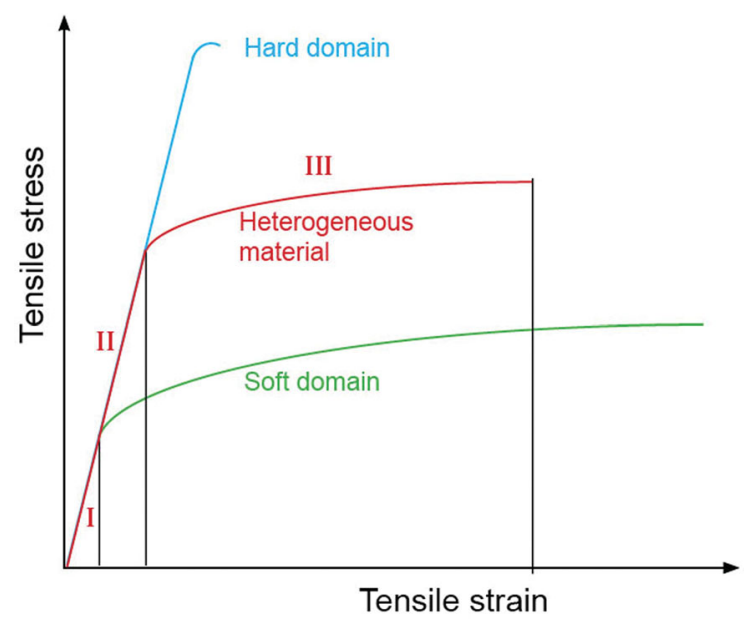

Figure 1. The three deformation stages of heterogeneous materials (the red stress-strain curve). (see Figure 1). In stage $I$, both soft and hard domains deform elastically, which is similar to a conventional homogeneous material.

In stage II, the soft domains will start dislocation slip first to produce plastic strain, while the hard domains will remain elastic, which creates a mechanical incompatibility. The soft domains need to deform together with the neighboring hard domains and, therefore, cannot plastically deform freely. The strain at the domain interface needs to be continuous, although the softer domains will typically accommodate more strains since they are plastically deforming. Therefore, there will be a plastic strain gradient in the soft domain near the domain interface. This strain gradient needs to be accommodated by geometrically necessary dislocations, which will make the softer phase appear stronger $[33,48]$, leading to synergetic strengthening to increase the global measured yield strength of the material [29].

In an extreme/ideal case, the soft domains are completely surrounded by the hard domain matrix so that the soft domain cannot change its shape as required by plastic deformation until the hard domain matrix starts to deform plastically. Geometrically necessary dislocations will pile-up at the domain boundaries in the soft domain, but cannot transmit across the domain boundary, building up high back-stress (see Figure 2). This can make the soft domain almost as strong as the hard domain matrix, making the global yield strength much higher than what is predicted by the rule of mixture [33].

In stage III, both the soft and hard domains deform plastically, but the soft domains sustain much higher strain than the hard domains, producing the socalled strain partitioning [43,49-53]. When neighboring domains sustain different plastic strains, strain gradients are expected to exist near the domain boundaries in both

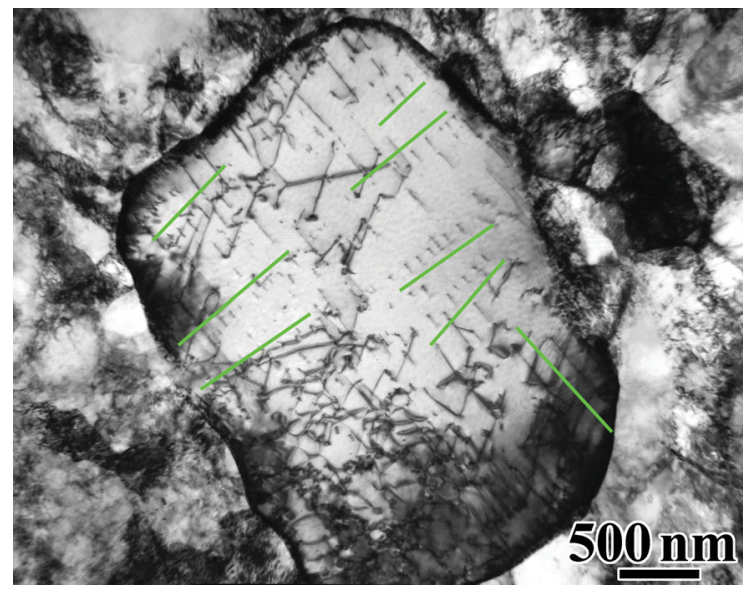

Figure 2. A 4- $\mu \mathrm{m}$ soft grain surrounded by a hard ultrafinegrained matrix in heterogeneous lamella Ti. Dislocation pile-ups are marked by green lines. 
the soft and hard domains. These strain gradients will become larger with increasing strain partitioning, and consequently produce back-stress work hardening. The back-stress work hardening will help with preventing necking during tensile testing, thus improving ductility. This is the primary reason why dual-phase steel has extraordinary work hardening, and consequently high ductility $[43,49,51,53]$.

\section{Back-stress strengthening and back-stress work hardening}

As discussed above, back-stress plays a significant role in the reported extraordinary strength and ductility of heterogeneous metals. Two types of dislocations are usually involved in the plastic deformation of metals and alloys: statistically stored dislocations and geometrically necessary dislocations. The flow stress as a function of dislocation density is conventionally calculated as [54-56]

$$
\tau=\alpha G b \sqrt{\rho_{\mathrm{S}}+\rho_{\mathrm{G}}},
$$

where $\tau$ is the shear flow stress, $\alpha$ is a constant, $G$ is the shear modulus, $b$ is the magnitude of Burgers vector, and $\rho_{\mathrm{S}}$ and $\rho_{\mathrm{G}}$ are the densities of statistically stored dislocations and geometrically necessary dislocations, respectively. In this equation, the statistically stored dislocations and geometrically necessary dislocations are treated to have the same contribution to the flow stress. Obviously, the back-stress caused by geometrically necessary dislocations area is ignored.

For conventional homogeneous metals, Equation (1) has been used to reasonably explain their mechanical behaviors, because the back-stress caused by the geometrically necessary dislocations is relatively small. However, for heterogeneous materials, the back-stress can be much higher than the strengthening associated with the statistically stored dislocations $[33,48]$, and therefore has to be considered. As discussed later, the back-stress can be utilized to design heterogeneous materials with unprecedented mechanical properties.

What is the physical origin of back-stress? To answer this question, let us have a look at the piling-up of geometric dislocations as schematically shown in Figure 3(A). Assume that there is a dislocation source at point $X$, which emits geometrically necessary dislocations with the same Burgers vector toward the domain boundary on a slip plane. Under an applied shear stress $\tau_{a}$, there are seven dislocations piled up and the system reached equilibrium. These dislocations collectively produce a long-range stress, $\tau_{b}$, toward the dislocation source as indicated by the arrow, which counterbalances the applied stress. The effective stress at the dislocation source can be expressed as $\tau_{e}=\tau_{a}-\tau_{b}$. If the critical
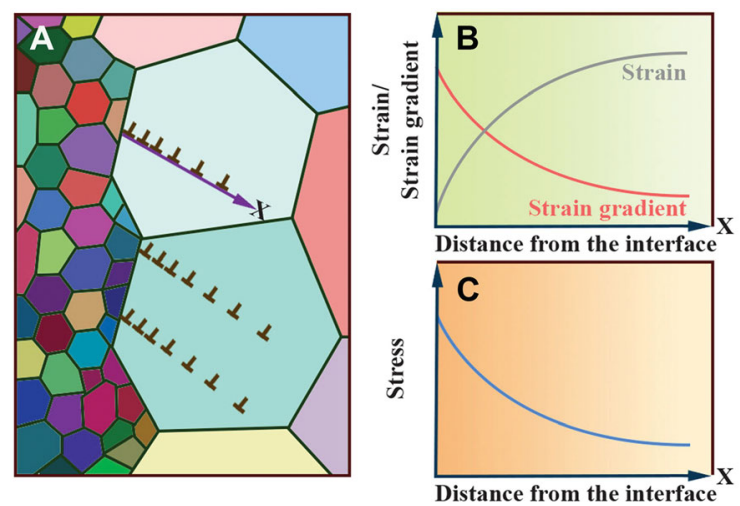

Figure 3. (A) Schematics of the piling-up of geometrically necessary dislocations. (B) Plastic strain and strain gradient as a function of distance from the domain interface. (C) The effective stress ( = applied stress - back-stress) as a function of distance from the domain interface.

stress to operate the dislocation source is $\tau_{c}$, then $\tau_{e}$ has to be higher than $\tau_{c}$ for the dislocation source to emit more dislocations. In other words, higher applied stress is needed for more dislocations to be piled up. Therefore, back-stress is a long-range stress created by geometrically necessary dislocations. The above discussion describes how back-stress can be produced from an individual dislocation pile. The experimentally measured back-stress is the global collective back-stress in the whole sample, just like the measured yield stress is a global stress contributed by individual yielding events in the whole sample.

Back-stress is connected with plastic strain gradient. The plastic strain is produced by the slip of dislocations, with each dislocation leaving a displacement of one Burgers vector in its wake. Therefore, in Figure 3(A) the strain is zero at the domain interface (pile-up head), and the strain is increased to seven Burges vector at the dislocation source. The black curve in Figure 3(B) shows a smoothed strain curve as a function of distance from the interface, the other curve in Figure 3(B) is the corresponding strain gradient curve. Therefore, the pile-up of geometrically necessary dislocations produces strain gradient as well as stress gradient (Figure $3(\mathrm{C})$ ). In other words, if strain gradient is observed, there will exist the pile-up of geometrically necessary dislocations and corresponding back-stress.

Note that another scenario to produce an array of geometrically necessary dislocations and the associated back-stress is when dislocations are emitted from a ledge on the domain boundary and/or grain boundary, but they form an array on a slip plane near the boundary [57]. This will produce the same back-stress to counterbalance the stress at the dislocation source, although the strain gradient will be different from what is described in Figure 3(B). 
The highest plastic strain always occurs at the dislocation source.

Back-stress has the same physical origin as the Bauschinger effect [58]. The larger Bauschinger effect corresponds to the higher back-stress. However, backstress can be used to improve the strength and ductility of metals if appropriate heterogeneous structures can be designed, instead of just a phenomenon of mechanical behavior as the Bauschinger effect is often regarded. The back-stress and its evolution during a tensile test can be measured experimentally $[33,48]$.

\section{Microstructural requirement for the optimum mechanical properties}

After discussing the role of back-stress in the mechanical behavior and the physical origin of back-stress, it naturally follows that we can design heterogeneous structures to maximize back-stress for the best mechanical properties. Since back-stress is produced by dislocation pile-up at domain boundaries, we should design the heterogeneous structure with high density of domain interfaces. However, the spacing between the domain interfaces should be large enough to allow an effective dislocation pile-up in at least the soft domains and ideally in both soft and hard domains. Another design factor is to maximize strain partitioning among heterogeneous domains, which will consequently increase the strain gradient, which will consequently increase back-stress work hardening. This means that the strength between the domains should be large, and the domain geometries should be such that large strain partitioning can be easily realized.

With the above two criteria for high back-stress, we can make comments on the effectiveness of various heterogeneous structures. For the gradient structure [27-32], there will be two dynamically migrating interfaces during the tensile tests $[28,29]$, which allows dislocation density accumulation over the whole sample volume. However, the low interface density also limits its capability of back-stress work hardening. For a bimodal structure $[10,25,34,35]$, the interface density is usually not maximized, which did not make the full use of the back-stress hardening potential. For a laminate structure $[39,40]$, the soft and hard laminates are subjected to the same applied strain, which limits their strain portioning capability and consequently back-stress development. For dual-phase steel [41-43], the hard martensitic domains typically account for $5-30 \%$ by volume and are embedded in the soft matrix. Although this allows significant strain portioning to increase the back-stress work hardening and consequently high ductility, the continuous soft matrix also allows the material to yield at low stress, which is why dual-phase materials typically have

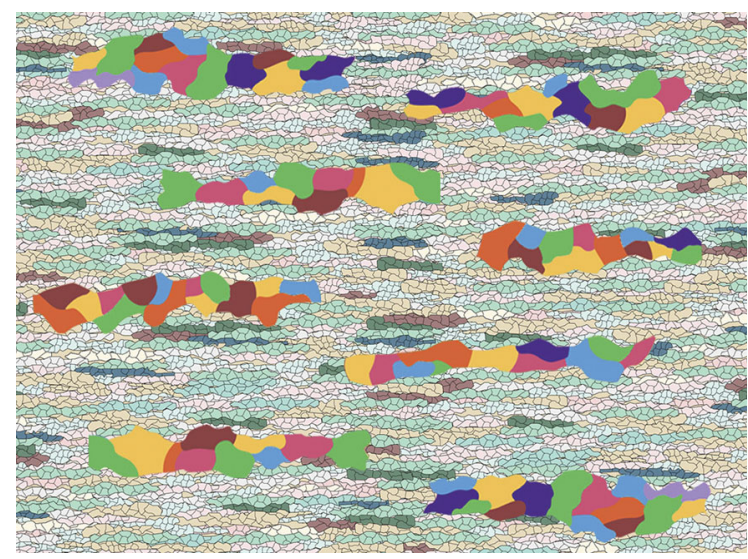

Figure 4. Schematics of lamella structure with elongated soft coarse-grained domains embedded in an ultrafine-grained matrix.

very high ductility, but limited enhancement in strength. For a harmonic structure [36-38], the soft domains are totally surrounded by hard domain layers similar to a cellular structure. It has been observed to enhance the ductility, but the strength improvement is so far limited, which could be improved by reducing the domain interface spacing and hard domain volume fraction to further constrain the soft domains. For the heterogeneous lamella structure [33], the soft lamella domains with a volume fraction of $<30 \%$ are embedded in a hard matrix (Figure 4), which renders it high strength because the rigid constraint by the hard matrix makes the soft domains almost as strong as the hard matrix during the deformation stage II (see Figure 1). The strong strain partitioning during deformation stage III also renders unprecedented high strain hardening, which increases its ductility. Therefore, the heterogeneous lamella structure [33] presents a near-ideal heterogeneous structure. This explains why the heterogeneous lamella structure has shown the most dramatic improvement in strength and ductility among all reported types of heterogeneous materials.

\section{Future perspective}

Heterogeneous materials is a fast emerging field that is to become a hot research field in the post-nanostructured materials era. There is a huge research community in the area of nanostructured materials, which has been extensively studied for over three decades. The maturing of this field and the challenge to the practical applications of nanostructured materials have made it hard to secure research funding in many countries in the world. Heterogeneous materials have many similarities to nanostructured materials because the hard domains could be 
nanostructured/ultrafine grained, which makes it easy for researchers in the nanostructured materials community to transit to the heterogeneous materials field. In addition, several types of heterogeneous materials can be produced by the current industrial processing technology so that their practical applications have a very low barrier.

There are many scientific and engineering issues that need to be addressed by both experimentalists and modelers from the communities of both materials science and mechanics. The heterogeneous materials community is quickly growing, with more international conferences and workshops being organized, for example, the biannual the minerals, metals \& materials society symposium on heterogeneous and gradient materials. Sessions on heterogeneous materials are also inserted into conventional successful symposia such as the TMS biannual meeting on ultrafine-grained materials. These activities help with the fast development in the area of heterogeneous materials.

\section{Disclosure statement}

No potential conflict of interest was reported by the authors.

\section{Funding}

X.W. was supported by the National Natural Science Foundation of China (NSFC) [grant number 11572328] and the Strategic Priority Research Program of the Chinese Academy of Sciences [grant number XDB22040503]. Y.Z. was supported by the US Army Research Office [grant number W911 NF-121-0009], and the Jiangsu Key Laboratory of Advanced Micro \& Nano Materials and Technology.

\section{References}

[1] Valiev RZ, Alexandrov IV, Zhu YT, et al. Paradox of strength and ductility in metals processed by severe plastic deformation. J Mater Res. 2002;17:5-8.

[2] Zhu YT, Liao XZ. Nanostructured metals - retaining ductility. Nature Mater. 2004;3:351-352.

[3] Jia D, Wang YM, Ramesh KT, et al. Deformation behavior and plastic instabilities of ultrafine-grained titanium. Appl Phys Lett. 2001;79:611-613.

[4] Zhao YH, Liao XZ, Horita Z, et al. Determining the optimal stacking fault energy for achieving high ductility in ultrafine-grained $\mathrm{Cu}-\mathrm{Zn}$ alloys. Mater Sci Eng A. 2008;493:123-129.

[5] Zhao YH, Bingert JF, Zhu YT, et al. Tougher ultrafine grain $\mathrm{Cu}$ via high-angle grain boundaries and low dislocation density. Appl Phys Lett. 2008;92:081903.

[6] Zhao YH, Zhu YT, Liao XZ, et al. Tailoring stacking fault energy for high ductility and high strength in ultrafine grained $\mathrm{Cu}$ and its alloy. Appl Phys Lett. 2006;89:121906.

[7] Wang YM, Ma E, Valiev RZ, et al. Tough nanostructured metals at cryogenic temperatures. Adv Mater. 2004;16:328-331.
[8] Zhao YH, Bingert JE, Liao XZ, et al. Simultaneously increasing the ductility and strength of ultra-fine-grained pure copper. Adv Mater. 2006;18:2949-2952.

[9] Zhao YH, Liao XZ, Cheng S, et al. Simultaneously increasing the ductility and strength of nanostructured alloys. Adv Mater. 2006;18:2280-2283.

[10] Zhao YH, Topping T, Bingert JF, et al. High tensile ductility and strength in bulk nanostructured nickel. Adv Mater. 2008;20:3028-3033.

[11] Cheng S, Zhao YH, Zhu YT, et al. Optimizing the strength and ductility of fine structured $2024 \mathrm{Al}$ alloy by nanoprecipitation. Acta Mater. 2007;55:5822-5832.

[12] Estrin Y, Vinogradov A. Extreme grain refinement by severe plastic deformation: A wealth of challenging science. Acta Mater. 2013;61:782-817.

[13] Meyers MA, Mishra A, Benson DJ. Mechanical properties of nanocrystalline materials. Prog Mater Sci. 2006;51:427-556.

[14] Lu L, Zhu T, Shen YF, et al. Stress relaxation and the structure size-dependence of plastic deformation in nanotwinned copper. Acta Mater. 2009;57:5165-5173.

[15] Wang YM, Ma E. Three strategies to achieve uniform tensile deformation in a nanostructured metal. Acta Mater. 2004;52:1699-1709.

[16] Yan FK, Liu GZ, Tao NR, et al. Strength and ductility of 316L austenitic stainless steel strengthened by nano-scale twin bundles. Acta Mater. 2012;60:1059-1071.

[17] Youssef K, Sakaliyska M, Bahmanpour H, et al. Effect of stacking fault energy on mechanical behavior of bulk nanocrystalline $\mathrm{Cu}$ and $\mathrm{Cu}$ alloys. Acta Mater. 2011;59:5758-5764.

[18] An XH, Han WZ, Huang CX, et al. High strength and utilizable ductility of bulk ultrafine-grained $\mathrm{Cu}-\mathrm{Al}$ alloys. Appl Phys Lett. 2008;92:201915.

[19] Zhang X, Wang H, Scattergood RO, et al. Tensile elongation (110\%) observed in ultrafine-grained $\mathrm{Zn}$ at room temperature. Appl Phys Lett. 2002;81:823-825.

[20] Huang XX, Kamikawa N, Hansen N. Increasing the ductility of nanostructured $\mathrm{Al}$ and Fe by deformation. Mater Sci Eng A. 2008;493:184-189.

[21] Wang GY, Li GY, Zhao L, et al. The origin of the ultrahigh strength and good ductility in nanotwinned copper. Mater Sci Eng A. 2010;527:4270-4274.

[22] Lu L, Shen YF, Chen XH, et al. Ultrahigh strength and high electrical conductivity in copper. Science. 2004;304:422-426.

[23] An XH, Wu SD, Zhang ZF, et al. Enhanced strengthductility synergy in nanostructured $\mathrm{Cu}$ and $\mathrm{Cu}-\mathrm{Al}$ alloys processed by high-pressure torsion and subsequent annealing. Scr Mater. 2012;66:227-230.

[24] Xiao GH, Tao NR, Lu K. Strength-ductility combination of nanostructured $\mathrm{Cu}-\mathrm{Zn}$ alloy with nanotwin bundles. Scr Mater. 2011;65:119-122.

[25] Wang YM, Chen MW, Zhou FH, et al. High tensile ductility in a nanostructured metal. Nature. 2002;419: 912-915.

[26] Zhu YT, Lowe TC, Langdon TG. Performance and applications of nanostructured materials produced by severe plastic deformation. Scripta Mater. 2004;51: 825-830.

[27] Lu K. Making strong nanomaterials ductile with gradients. Science. 2014;345:1455-1456. 
[28] Wu XL, Jiang P, Chen L, et al. Extraordinary strain hardening by gradient structure. Proc Natl Acad Sci USA. 2014;111:7197-7201.

[29] Wu XL, Jiang P, Chen L, et al. Synergetic strengthening by gradient structure. Mater Res Lett. 2014;2:185-191.

[30] Fang TH, Li WL, Tao NR, et al. Revealing extraordinary intrinsic tensile plasticity in gradient nano-grained copper. Science. 2011;331:1587-1590.

[31] Chen AY, Liu JB, Wang HT, et al. Gradient twinned 304 stainless steels for high strength and high ductility. Mater Sci Eng A. 2016;667:179-188.

[32] Wei YJ, Li YQ, Zhu LC, et al. Evading the strength- ductility trade-off dilemma in steel through gradient hierarchical nanotwins. Nat Commun. 2014;5:3580.

[33] Wu XL, Yang MX, Yuan FP, et al. Heterogeneous lamella structure unites ultrafine-grain strength with coarsegrain ductility. Proc Natl Acad Sci USA. 2015;112:1450114505.

[34] Han BQ, Huang JY, Zhu YT, et al. Strain rate dependence of properties of cryomilled bimodal $5083 \mathrm{Al}$ alloys. Acta Mater. 2006;54:3015-3024.

[35] Han BQ, Lee Z, Witkin D, et al. Deformation behavior of bimodal nanostructured $5083 \mathrm{Al}$ alloys. Metall Mater Trans A. 2005;36:957-965.

[36] Sawangrat C, Kato S, Orlov D, et al. Harmonic-structured copper: performance and proof of fabrication concept based on severe plastic deformation of powders. J Mater Sci. 2014;49:6579-6585.

[37] Zhang Z, Vajpai SK, Orlov D, et al. Improvement of mechanical properties in SUS304L steel through the control of bimodal microstructure characteristics. Mater Sci Eng. 2014;598:106-113.

[38] Vajpai SK, Ota M, Watanabe T, et al. The development of high performance Ti-6Al-4V alloy via a unique microstructural design with bimodal grain size distribution. Metall Mater Trans A. 2015;46:903-914.

[39] Ma XL, Huang CX, Moering J, et al. Mechanical properties in copper/bronze laminates: role of interfaces. Acta Mater. 2016;116:43-52.

[40] Beyerlein IJ, Mayeur JR, Zheng SJ, et al. Emergence of stable interfaces under extreme plastic deformation. Proc Natl Acad Sci USA. 2014;111:4386-4390.

[41] Calcagnotto M, Adachi Y, Ponge D, et al. Deformation and fracture mechanisms in fine- and ultrafine-grained ferrite/martensite dual-phase steels and the effect of aging. Acta Mater. 2011;59:658-670.

[42] Li ZM, Pradeep KG, Deng Y, et al. Metastable highentropy dual-phase alloys overcome the strength-ductility trade-off. Nature. 2016;534:227-231.
[43] Park K, Nishiyama M, Nakada N, et al. Effect of the martensite distribution on the strain hardening and ductile fracture behaviors in dual-phase steel. Mater Sci Eng. 2014;604:135-141.

[44] Wu XL, Yuan FP, Yang MX, et al. Nanodomained nickel unite nanocrystal strength with coarse-grain ductility. Sci Rep. 2015;5:11728.

[45] Lu K, Yan FK, Wang HT, et al. Strengthening austenitic steels by using nanotwinned austenitic grains. Scr Mater. 2012;66:878-883.

[46] Li YS, Tao NR, Lu K. Microstructural evolution and nanostructure formation in copper during dynamic plastic deformation at cryogenic temperatures. Acta Mater. 2008;56:230-241.

[47] Ma E, Zhu T. Towards strength-ductility synergy through the design of heterogeneous nanostructures in metals. Mater Today. 2017; doi:10.1016/ j.mattod.2017.02.003

[48] Yang MX, Pan Y, Yuan FP, et al. Back stress strengthening and strain hardening in gradient structure. Mater Res Lett. 2016;4:145-151.

[49] Cong ZH, Jia N, Sun X, et al. Stress and strain partitioning of ferrite and martensite during deformation. Metall Mater Trans A. 2009;40:1383-1387.

[50] Tasan CC, Diehl M, Yan D, et al. Integrated experimentalsimulation analysis of stress and strain partitioning in multiphase alloys. Acta Mater. 2014;81:386-400.

[51] Han QH, Asgari A, Hodgson PD, et al. Strain partitioning in dual-phase steels containing tempered martensite. Mater Sci Eng. 2014;611:90-99.

[52] Wang MM, Tasan CC, Ponge D, et al. Nanolaminate transformation- induced plasticity-twinning-induced plasticity steel with dynamic strain partitioning and enhanced damage resistance. Acta Mater. 2015;85:216-228.

[53] Yang MX, Yuan FP, Xie QG, et al. Strain hardening in Fe$16 \mathrm{Mn}-10 \mathrm{Al}-0.86 \mathrm{C}-5 \mathrm{Ni}$ high specific strength steel. Acta Mater. 2016;109:213-222.

[54] Gao H, Huang Y, Nix WD, et al. Mechanism-based strain gradient plasticity - I. Theory. J Mech Phys Solids. 1999;47:1239-1263.

[55] Gao HJ, Huang YG. Geometrically necessary dislocation and size-dependent plasticity. Scr Mater. 2003;48:113-118.

[56] Ashby MF. Deformation of plastically non-homogeneous materials. Philos Mag. 1970;21:399-424.

[57] Murr LE. Dislocation ledge sources: dispelling the myth of frank-read source importance. Metall Mater Trans A. 2016;47:5811-5826.

[58] Kato H, Moat R, Mori T, et al. Back stress work hardening confirmed by Bauschinger effect in a TRIP steel using bending tests. ISIJ Int. 2014;54:1715-1718. 